\section{Quantitative Changes in $\gamma$-Aminobutyric Acid Induced by Low Temperature in Rice Plants}

Among the free amino-acids occurring in higher plants increasing attention is being paid to $\gamma$-aminobutyric acid, although it has not been identified as a constituent of proteins. The significance of this compound in the nitrogen metabolism of plants is clearly indicated by the work of Steward and collaborators ${ }^{1,2}$, and also by our recent studies on rice varieties exhibiting various degrees of resistance against the 'browning disease' of rice ('brusone,' 'aki-ochi').

Our experimental material consisted of the brusoneresistant Precoce Allorio and brusone-susceptible Dunghan Shali rice varieties. The experiments were carried out in the field on limeless soils. The shoots of rice plants were removed shortly after flowering, the exudate yielded by guttation was collected and analysed for free amino-acids by paper chromatography ${ }^{3}$ in a solvent system containing butanol, acetic acid, and water in the proportions $2: 1: 1 . \quad \gamma$-Aminobutyric acid was not detected in the exudate of Dunghan Shali (susceptible variety) for some days after a period at a low temperature $\left(12-14^{\circ} \mathrm{C}\right.$.) and of unfavourable light relation. A similar decrease in $\gamma$-aminobutyric acid did not occur in Precoce Allorio which is regarded as a variety resistant to brusone. The content of $\gamma$-aminobutyric acid of both varieties was similar and constant in rice plants kept at normal temperatures $\left(20-22^{\circ} \mathrm{C}\right.$.). The experiments were repated several times with the same results (Fig. 1).

When, due to bad weather, the temperature of the inundation water and of the soil drops, the incidence of disease is higher. Therefore, low temperature is regarded as one of the main factors increasing the susceptibility of rice plant to brusone ${ }^{4}$. It seems possible that the detection of resistant varieties by

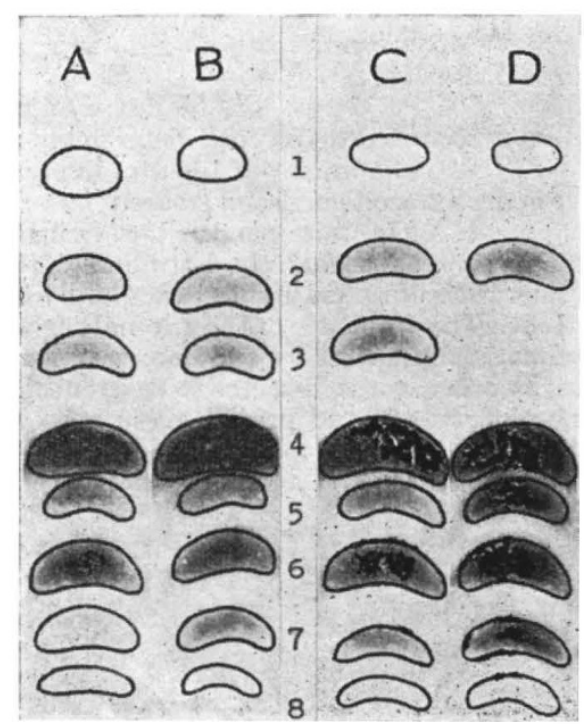

Fig. 1. A, Precoce Allorio (control); B, Precoce Allorio (low temperaFig. 1. A, Precoce Allorio (control); B, Precoce Allorio (low tempera-
ture); $C$, Dunghan Shali (control); D, Dunghan Shall (low tempera-
ture).

1 , leucine; 2 , valine; $3, \gamma$-aminobutyric acid; 3 , alanine; 5 , glutamic acid; 6 , aspartic acid + serine + giutamine; 7 , histidine + arginine; 8 , cyst(e)ine means of some chemical characteristics will yield a reasonable tool which might substitute the long and empirical work of plant breeders.

\section{F. Zsoldos}

Institute of Plant Physiology,

University of Szeged,

Szeged, Hungary.

$$
\text { April } 6 .
$$

I Steward, F. C., and Thompson, J. F., Nature, 171, 1063 (1953). 2 Steward, F. C., Bidwell, R, G. S., and Yemm, I. W., J. Exp. Bot., 9, 11 $(1958)$

Matthias, W., Naturwiss., 41, 17 (1954) Rice Monograph (Japan, 1955).
.

\section{BIOLOGY}

\section{Plastron Respiration in the Eggs of Drosophila and other flies}

Ir has long been known that the conspicuous projections near the anterior end of the eggs of Scopeuma, Drosophila, and other flies are concerned in respiration, and the projections have been called respiratory horns. The site of entry of oxygen into the horn has not previously been determined, but where not explicitly stated it has been implied that oxygen enters through holes in the distal end of the horn. Reaumur ${ }^{1}$ regarded the respiratory horns of Scopeuma as floats that served to prevent the submergence of the eggs and so their asphyxiation. A similar function has been postulated for the respiratory horns of Drosophila by Wigglesworth and Beament ${ }^{2}$. However, the eggs of Scopeuma stercorarium L. and Drosophila mealnogestar Meig., as well as those of many other species with similar respiratory horns, are heavier than water even when the chorion and plastron are air-filled and besides are normally stuck to the substrate: they do not float if submerged, when under natural conditions they might be washed away from the larval food supply. Portions of cow pats containing eggs of Scopeuma and Hebecnema umbratica Meig. were repeatedly submerged in water, but the eggs were never detached. Of course these eggs and those of Drosophila and other species can be suspended from the surface film if they are freed from their attachment to the substrate and a line of contact with the water and air is established. Under these conditions their centres and buoyancy and gravity are such that the tips of the respiratory horns often project above the surface film.

The term plastron has been restricted to describe a gas film of constant volume and an extensive waterair interface. Such films are held in position by a system of hydrofuge structures and are capable of resisting water under pressure. In well-aerated water a plastron enables the insect to remain immersed indefinitely, when it obtains the oxygen it requires from the ambient water. What was known of plastron respiration in insects was summarized by Thorpe ${ }^{3}$ in 1950. Since then the plastron method of respiration has been found in a variety of insect pup $x^{4,5}$ and now in the eggs of Sepsis violacea Meig., Drosophila melano. gaster Meig. and other species of the genus, Musca autumnalis Deg., Hebecnema umbracata Meig., Scopeuma stercorarium $\mathbf{L}$., and other flies.

A detailed account of the structure of the respiratory horns of the eggs of Drosophila, Scoperma and other flies will be published elsewhere. In all the 